\title{
Ocorrência de moscas ectoparasitas (Diptera, Streblidae e Nycteribiidae) de morcegos (Mammalia, Chiroptera) no Cerrado de Brasília, Distrito Federal, Brasil ${ }^{1}$
}

\author{
Gustavo Graciolli ${ }^{2}$ \\ Ludmilla S. Aguiar ${ }^{3}$
}

\begin{abstract}
Records of batflies (Diptera, Streblidae and Nycteribiidae) on bats (Mammalia, Chiroptera) from the Cerrado of Brasília, Distrito Federal, Brazil. Two species of Basilia Miranda Ribeiro, 1903 (Nycteribiidae) and eigth species of Streblidae were collected from Brasília. Basilia anceps Guimarães \& D'Andretta, 1956; B. hughscotti Guimarães \& D'Andretta, 1956; Megistopoda aranea (Coquillet, 1899); M. proxima (Séguy, 1926) and Neotrichobius delicatus (Machado-Allison, 1966) are collected for the first time at the Federal District.

KEY WORDS. Streblidae, Nycteribiidae, Batflies, Cerrado biome, ectoparasites
\end{abstract}

Streblidae e Nycteribiidae (Diptera, Hippoboscoidea) são dípteros hematófagos ectoparasitos exclusivos de morcegos. Na Região Neotropical são encontrados parasitando morcegos das famílias Phyllostomidae, Mormoopidae, Noctilionidae, Vespertilionidae, Natalidae, Thyropteridae e Molossidae. No Brasil, até o momento foram registradas 20 espécies de Streblidae pertencentes a sete gêneros (COIMBRA et al. 1984; GraCiOlli \& COELHO 2001) infestando morcegos Phyllostomidae no Distrito Federal. Espécies de Nycteribiidae são, até o presente, inéditas nesta região.

Esse trabalho foi realizado no Jardim Botânico do Distrito Federal (1553' 17'S, 47 50 '46"W), uma reserva distrital com cerca de 4.500 ha, e na Reserva do Roncador ( $\left.15^{\circ} 55^{\prime} 4^{\prime \prime} \mathrm{S}, 4^{\circ} 53^{\prime} 11^{\prime \prime} \mathrm{W}\right)$, uma unidade de conservação de 1.360 ha. Essas duas áreas somam-se à Fazenda Água Limpa e áreas adjacentes para formar a Área de Proteção Ambiental Cabeça do Veado, uma unidade de conservação com aproximadamente 25.000 ha. Esse é o mais importante conjunto de áreas protegidas ao sul da cidade de Brasília.

Os morcegos foram coletados na mata de galeria e no cerrado sensu strictu. A mata de galeria é uma formação florestal perenifólia ou mesofítica que ocorre tipicamente ao longo dos cursos d'água, com largura variando em função das características edáficas e topográficas (RIBEIRO \& WALTER 1998). Junto com o cerradão, é a fisionomia que tem maior complexidade estrutural no bioma, podendo existir até três estratos diferenciados. O cerrado pode ser caracterizado como uma formação savânica aberta (woodland savana) onde a cobertura vegetal está entre 20-80\% (RIBEIRO \& WALTER 1998; WALTER \& SAMPAIO 1998). O estrato inferior é coberto por gramíneas e diversas espécies de porte herbáceo.

1) Contribuição número 1349 do Departamento de Zoologia, Universidade Federal do Paraná.

2) Departamento de Zoologia, Universidade Federal do Paraná. Caixa Postal 19020, 81531-980 Curitiba, Paraná, Brasil. E-mail: mingau@bio.ufpr.br

3) Embrapa Cerrados. Rodovia Brasília-Fortaleza BR 020, Km 18, Planaltina, Distrito Federal, Brasil, 73301-970. E-mail: ludmilla@cpac.embrapa.br 
Os morcegos foram capturados a partir de abril de 1998 até março de 1999 , durante 18 dias consecutivos a cada mês, em quatro áreas de cerrado sensu strictu e em duas áreas de mata de galeria. Foram utilizadas 10 redes de neblina (mist nets) (ATX $12 \times 3$ m e malha de $35 \mathrm{~mm}$ ) abertas em cada um dos sítios das 18 às 23 horas. A cada 20 minutos as redes eram inspecionadas para coleta dos indivíduos capturados. Espécimes testemunhos foram depositados na coleção do Departamento de Zoologia da Universidade de Brasília. Os morcegos encontrados com altas infestações tiveram os ectoparasitos coletados e fixados em álcool a 70\% para identificação. Os espécimes foram depositados na Coleção de Entomologia Pe J.S. Moure, Departamento de Zoologia, Universidade Federal do Paraná (DZUP).

Foram encontradas duas espécies de um gênero de Nycteribiidae e sete espécies de quatro gêneros de Streblidae. Estes são os primeiros registros de moscas da família Nycteribiidae no Distrito Federal. Quatro novas espécies da família Streblidae foram registradas, aumentando para 24 o número de representantes desta família no bioma Cerrado.

O material examinado por família, contendo as respectivas espécies de dípteros e de hospedeiros, bem como alguns comentários pertinentes são apresentados a seguir.

\section{Nycteribiidae}

\section{Basilia anceps Guimarães \& D'Andretta, 1956}

Material examinado. Um macho e 1 fêmea sobre Myotis nigricans (Schinz, 1821), L.S. Aguiar leg., 21/VII/1998; 1 macho e 1 fêmea sobre Myotis riparius Handley, 1960, L.S. Aguiar leg., 27/IX/1998.

Comentários. Espécie pertencente ao grupo juquiensis (THEODOR 1967). Novo registro para o Brasil. Anteriormente, Basilia anceps havia sido coletada no Panamá, sobre Myotis nigricans e Myotis simus Thomas, 1901 (GUIMARÃES 1966); na Colômbia, sobre $M$. nigricans (GUIMARÃES \& D'ANDRETTA 1956) e no Peru, sobre $M$. nigricans e Myotis riparius (GUIMARÃES \& D'ANDRETTA 1956; GUERRERO 1996).

\section{Basilia hughscotti Guimarães, 1946}

Material examinado. Duas fêmeas sobre Myotis nigricans, L.S. Aguiar leg., 21/VII/1998; 1 fêmea sobre M. nigricans, L.S. Aguiar leg., 25/VII/1998; 2 machos e 1 fêmea sobre Myotis riparius, L.S. Aguiar leg., 27/IX/1998; 1 fêmea sobre Eptesicus furinalis (d'Orbygny, 1847), L.S. Aguiar leg., 19/X/1998; 1 fêmea sobre E. furinalis, L.S. Aguiar leg., 26/XI/1998.

Comentários. Espécie pertencente ao grupo speiseri (THEODOR 1967). Novo registro para o Distrito Federal. Esta espécie foi descrita por GUIMARÃES (1946) a partir de uma fêmea coletada sobre o morcego filostomídeo Chrotopterus auritus (Peters, 1856) no Rio Grande do Sul. Apenas o holótipo era conhecido. Os espécimens examinados foram comparados com o holótipo de B. hughscotti, o qual se encontra em péssimo estado de conservação, e com a descrição original. Nosso dados indicam a baixa especificidade de Basilia hughscotti, que parasita, além do hospedeiro-tipo, três espécies de morcegos da família Vespertilionidae. 


\section{Streblidae}

\section{Aspidoptera falcata Wenzel, 1976}

Material examinado. Um fêmea sobre Sturnira lilium (E. Geoffroy, 1810), L.S. Aguiar leg., 24/VII/1998; 1 fêmea sobre S. lilium, L.S. Aguiar leg., 26/VIII/ 1998; 1 macho e 1 fêmea sobre Artibeus jamaicensis Leach, 1821, L.S. Aguiar leg., 18/X/1998; 1 macho e 2 fêmeas sobre S. lilium, L.S. Aguiar leg., 26/XI/1998; 1 macho sobre S. lilium, L.S. Aguiar leg., 27/XI/1998; 2 fêmeas sobre Artibeus lituratus (Olfers, 1818) L.S. Aguiar leg., 15///1999.

Comentário. Novo registro para o Distrito Federal. Duas fêmeas examinadas estavam parasitadas por uma espécie de fungo Laboulniomycetes. Os talos do fungo estavam localizados no dorso do abdômen e dos fêmures.

\section{Megistopoda aranea (Coquillett, 1899)}

Material examinado. Um macho sobre Glossophaga soricina (Pallas, 1767), L.S. Aguiar leg., 30/IX/1998.

Comentário. Novo registro para o Distrito Federal. Megistopoda aranea normalmente parasita morcegos do gênero Artibeus Gray, 1838 (GUERRERO 1994), portanto o registro sobre $G$. soricina deve ser acidental ou secundário.

\section{Megistopoda proxima (Séguy, 1926)}

Material examinado. Um macho sobre Sturnira lilium, L.S. Aguiar leg., 20/VII/1998; 1 fêmea sobre S. lilium, L.S. Aguiar leg., 19/VIII/1998; 1 fêmea sobre S. lilium, L.S. Aguiar leg., 20/VIII/1998; 1 fêmea sobre S. lilium, L.S. Aguiar leg., 26/VIII/1998; 1 macho sobre S. lilium, L.S. Aguiar leg., 08/IX/1998; 1 macho sobre S. lilium, L.S. Aguiar leg., 21/X/1998; 1 macho sobre S. lilium, L.S. Aguiar leg., 16/XI/1998; 1 macho e 1 fêmea sobre $S$. lilium, L.S. Aguiar leg., 26/XI/1998; 1 fêmea sobre S. lilium, L.S. Aguiar leg., 27/XI/1998.

Comentário. Novo registro para o Distrito Federal. Espécie com ampla distribuição geográfica, sendo encontrada deste o sul do México ao Uruguai sobre espécies de Sturnira Gray, 1842, principalmente S. lilium (GUERRERO 1994).

\section{Neotrichobius delicatus (Machado-Allison, 1966)}

Material examinado. Um macho sobre Artibeus cinereus (Gervais, 1856), L.S. Aguiar leg., 23/III/1999; 1 fêmea sobre A. cinereus, L.S. Aguiar leg., 24/II/1999.

Comentário. Novo registro para o Distrito Federal. Uma vez que GUERRERO (1994) havia colocado em dúvida a ocorrência de Neotrichobius delicatus no Brasil, a sua presença é presentemente confirmada.

\section{Paratrichobius longicrus (Miranda Ribeiro, 1907)}

Material examinado. Duas fêmeas sobre Platyrrhinus lineatus (E. Geoffroy, 1810), L.S. Aguiar leg., 13/VII/1998; 1 macho e 1 fêmea sobre P. lineatus, L.S. Aguiar leg., 23/XI/1998; 1 fêmea sobre Artibeus lituratus, L. Aguiar leg., 10/XII/ 1998; 2 machos e 1 fêmea sobre A. lituratus, L.S. Aguiar leg., 26/III/1999; 1 macho e 1 fêmea sobre A. lituratus, L.S. Aguiar leg., 26/IIU/1999; 1 macho sobre A. lituratus, L.S. Aguiar leg., 17/IV/1999. 
Comentário. Previamente no Distrito Federal, CoIMBRA et al. (1984) registraram $P$. longicrus sobre A. lituratus. No estado de Minas Gerais, KOMENO \& LinHARES (1999) encontraram em área de cerrado $P$. longicrus parasitando $P$. lineatus.

\section{Trichobius joblingi Wenzel, 1966}

Material examinado. Uma fêmea sobre Carollia perspicillata (Linnaeus, 1758), L.S. Aguiar leg., 29/VII/1998; 1 fêmea sobre C. perspicillata, L.S. Aguiar leg., 10/IX/1998; 1 macho sobre A. lituratus, L.S. Aguiar leg., 04/II/1999; 3 machos e 2 fêmeas sobre C. perspicillata, L.S. Aguiar leg., 17/IV/1999.

Comentário. Anteriormente, CoIMBRA et al. (1984) haviam assinalado esta mesma infestação na mesma região.

\section{Trichobius Ionchophyllae Wenzel, 1966}

Material examinado. Dois machos e 1 fêmea sobre Lonchophylla dekeyseri Taddei, Vizoto \& Sazima, 1983, L.S. Aguiar leg., 24/IV/1999.

Comentário. Espécie anteriormente registrada no Distrito Federal por GRACIOLLi \& COELHO (2001) sobre a mesma espécie de hospedeiro.

\section{Trichobius sp.}

Material examinado. Uma fêmea sobre Platyrrhinus lineatus, L.S. Aguiar leg., 20/VI/1998; 2 machos sobre P. lineatus, L. Aguiar leg., 09/IX/1998.

Comentário. Espécie do complexo dugesi (WENZEL et al. 1966). Provavelmente se trate de Trichobius angulatus Wenzel, 1976, no entanto, o mau estado de conservação dos exemplares não permite uma identificação precisa.

\section{REFERÊNCIAS BIBLIOGRÁFICAS}

Coimbra JR., C.E.A.; L.R. Guimarães \& D.A. Mello. 1984. Ocorrência de Streblidae (Diptera: Pupipara) em morcegos capturados em regiões de cerrado do Brasil Central. Revta bras. Ent. 28: 547-550.

Graciolli, G. \& D.C. Coelho. 2001. Streblidae (Díptera, Hippoboscoidea) sobre morcegos filostomídeos (Chiroptera, Phyllostomidae) em cavernas do Distrito Federal, Brasil. Revta bras. Zool. 18 (3): 965-970.

Guerrero, R. 1994. Catalogo de los Streblidae (Diptera: Pupipara) parasitos de murcielagos (Mammalia: Chiroptera) del Nuevo Mundo. IV. Trichobiinae com alas desarrolladas. Bol. Entomol. Venez., n.s., 9: $161-192$.

— 1996. Streblidae (Diptera: Pupipara) parásitos de los murciélagos de Pakitza, Parque Nacional Manu (Perú), p. 627-641. In: D.E. Wilson \& A. SAndoval (Eds). MANU: La biodiversidad del sureste del Perú. Washington, DC, Smithsonian Institution, 679p.

GUIMARÃES, L.R. 1946. Revisão das espécies Sul-americanas do gênero Basilia (Diptera-Nycteribiidae). Arq. Zool., São Paulo, 5: 1-88.

1966. Nycteribiid batflies from Panama (Diptera: Nycteribiidae), p. 393-404. In: R.L. Wenzel \& V.J. TiPTon (Eds). Ectoparasites of Panama. Chicago, Field Mus. Nat. Hist., XII+861p. GuimarÃes, L.R. \& M.A.V. D’AndRETTA. 1956. Sinopse dos Nycteribiidae (Diptera) do Novo Mundo. Arq. Zool., São Paulo, 10: 1-184.

Komeno, C.A. \& A.X. LinHARES. 1999. Batflies parasitic on some phyllostomid bats in Southeastern Brazil: Parasitism rates and host-parasite relationships. Mem. Inst. Oswaldo Cruz 94: 151-156.

Ribeiro, J.F. \& B.M.T. WALTER. 1998. Fitofisionomias do Bioma Cerrado, p. 89-166. In: S.M. SANo \& S.P. DE AlmeIDA (Eds). Cerrado: ambiente e flora. Brasília, EMBRAPA-CPAC, 250p. 
THEODOR, O. 1967. An illustrated catalogue of the Rothschild collection of Nycteribiidae (Diptera) in the British Museum (Natural History) with keys and the short descriptions for the identification of subfamilies, genera, species and subspecies. Brit. Mus. (Nat. Hist.) Publ. 665: 1-506.

Walter, B.M.T. \& A.B. SAmpaio. 1998. A vegetação da Fazenda Sucupira. Brasília, EMBRAPA, Recursos Genéticos e Biotecnologia, 76p.

WenZeL, R.L.; V.J. TIPTON \& A. Kiewlicz. 1966. The streblid batflies of Panama (Diptera: Calyptera: Streblidae). Pp. 405-675 in R.L. Wenzel \& V.J. TiPTon (Eds). Ectoparasites of Panama. Chicago, Field Mus. Nat. Hist., XII+861p.

Recebido em 07.XII.2001; aceito em 24.VI.2002. 